\title{
EL FILÓLOGO PEDRO URBANO GONZÁLEZ DE LA CALLE (1879-1966). UNA APROXIMACIÓN A SU VIDA Y OBRA
}

\author{
THE PHILOLOGIST PEDRO URBANO GONZÁLEZ \\ DE LA CALLE (1879-1966). \\ AN APPROACH TO HIS LIFE AND WORK
}

\author{
Santos M. Protomártir Vaquero \\ Universidad Nacional de Educación a Distancia \\ santos.protomartir@gmail.com
}

REsumen: En este trabajo se esboza la carrera del filólogo y humanista español Pedro Urbano González de la Calle (1879-1966), desde sus años como catedrático de Filología Latina de la Universidad de Salamanca y de la Universidad Central de Madrid hasta su experiencia como exiliado en Colombia y México. Se destaca la importancia de sus trabajos primeros sobre Francisco Sánchez de las Brozas "El Brocense" y otros humanistas del Renacimiento español, así como sus investigaciones posteriores relacionadas con la obra del lingüista colombiano Rufino J. Cuervo. Cierra el estudio una lista de sus principales publicaciones.

Palabras clave: González de la Calle; filología; exilio español; humanismo;

Colombia; México.

Abstract: This paper traces the career of the Spanish philologist Pedro Urbano González de la Calle (1879-1966), from his early years as professor of Latin Philology at the University of Salamanca and the Central University of Madrid through to his later experience as an exile living in Colombia and Mexico. Importance is placed on his early work on Francisco Sánchez de las Brozas "El Brocense" and other Spanish Renaissance humanists, as well as on his subsequent research relating to the work of the Colombian linguist Rufino J. Cuervo. The paper closes with a list of his principal publications.

Keywords: González de la Calle; philology; Spanish exile; humanism; Colom-

bia; México.

Recepción: 6 de julio de 2016; aceptación: 25 de abril de 2017.

D.R. () 2018. Nueva Revista de Filología Hispánica

Licencia Creative Commons Attribution-NonCommercial (CC BY-NC) 4.0 International 
En 1967, Thesaurus, el Boletín del Instituto Caro y Cuervo de Bogotá, publicó una larga y sentida necrológica sobre el filólogo Pedro Urbano González de la Calle, firmada por el presidente y el secretario del mismo instituto, Gabriel Betancur Mejía y Francisco Sánchez Arévalo (1967, pp. 131-144). Además de lamentar el fallecimiento del ilustre extremeño, los autores de la nota exaltan sus méritos como maestro, investigador y humanista, y lo elogian por su ejemplar dedicación a la filología y a la lingüística y, muy especialmente, a los estudios de lengua y literatura españolas. Y, en efecto, a lo largo de sus sesenta años de vida profesional, González de la Calle fue dejando una obra de indudable trascendencia filológica, primero en España y después en Colombia y México, tal y como vamos ahora a comentar. Si bien sólo podemos detenernos en algunos de sus trabajos más importantes, al final de este artículo ofrecemos una bibliografía más completa.

\section{LOS PRIMEROS AÑOS. ESPAÑA}

Pedro Urbano González de la Calle nació en Madrid el 12 de noviembre de 1879 en el seno de una familia extremeña. Después de estudiar el bachillerato en el Instituto San Isidro, donde su padre, Urbano González Serrano, era catedrático de filosofía y un importante pensador krausista, Pedro Urbano cursó filosofía y letras en la Universidad Central; ahí obtuvo los grados de licenciado con nota de sobresaliente y, más tarde, en 1904, el grado de doctor con Premio Extraordinario, con una tesis sobre el humanista sevillano Sebastián Fox Morcillo. A raíz de estos éxitos, con apenas veinticinco años de edad, pasó a ser catedrático de Lengua y Literatura Latinas de la Universidad de Salamanca ${ }^{1}$. Allí coincidió, entre otros, con Miguel de Unamuno, a quien prestó su apoyo tras su destitución como rector en 1914. Fruto de estas relaciones de compañerismo y amistad es el artículo que González de la Calle publicaría en 1941 en la Revista Hispánica Moderna, titulado "Recuerdos personales de la vida profesional del maestro Unamuno".

A estos años salmantinos corresponden trabajos como los dedicados a Juan de Mariana y a Benito Arias Montano, a autores latinos como Catulo, Marcial o Virgilio, a temas de lingüística latina y a asuntos históricos como su investigación sobre "Oposiciones a cátedras en la Universidad de Salamanca durante el primer decenio de la segunda mitad del siglo xvi (1550 a 1560). Ensayo monográfico”, publicado en Madrid en 1933. Pero sin duda, el trabajo más importante de este primer periodo de su carrera como filólogo fue la monogra-

1 González de la Calle ingresó en el cuerpo de catedráticos por oposición libre el 10 de mayo de 1904. 
fía que dedicó al Brocense. Cabe señalar que una primera aproximación a esta figura fue el texto que leyó con motivo de la inauguración del curso universitario de 1912. Diez años después dio a la imprenta una monografía más elaborada, su Ensayo biográfico. Vida profesional y académica de Francisco Sánchez de las Brozas (1922), que pronto se convirtió en una referencia obligada para todo estudioso del Brocense y, en general, para toda persona interesada en el humanismo español. A lo largo de sus páginas, González de la Calle desmenuzó puntualmente el historial académico y profesional de su predecesor y paisano. Para ello, consultó los libros de actas del claustro, el de la matrícula de alumnos, así como toda la documentación obrante en la Universidad que permitiera seguir la huella del humanista. El estudio de estos documentos también le proporcionó materiales para otra investigación paralela, que publicaría en 1933 bajo el título de Oposiciones a cátedras en la Universidad de Salamanca durante el primer decenio de la segunda mitad del XVI. Allí abordó también todos los temas clave de la doctrina gramatical del Brocense: la división de la gramática, su teoría de la oración simple y sus constituyentes; la elipsis como uno de los puntos clave de su concepción gramatical, así como la división tripartita de nomina, verba y particulae.

Al tratar estas y otras cuestiones, Pedro Urbano mostró admiración y devoción hacia la figura del Brocense, con quien se sentía identificado por su honestidad y por la forma científica con que exponía sus ideas. De hecho, una afirmación del maestro de Retórica la adopta como lema suyo: "Non... dubium est, quin rerum omnium, etiam vocum, reddenda sit ratio, quam si ignoravimus rogati, fateamur potius nos nescire quam nullam esse constanter affirmare" (F. Sanctii Brocensis, Minerva sive de causis linguae latinae, cap. 1, f. 6). Otra cuestión que el filólogo estudió fue la actitud del Brocense hacia Antonio de Nebrija, la sustitución de su Gramática latina en la Universidad y el célebre prólogo a la Minerva, de la que destacó sobre todo el largo párrafo que va del 4, Nihil omnino hic novi afferimus: stulti vulgo ride sermonem, sed antiquitatem, quae barbarorum (Mammotrectos dico, Catholicones et Pastranas) saevitia iacuerat, sublevamus et in gradum reponimus... al 19: Quid si illud interim virgilianum occinebat: Exoriare aliquis nostris ex ossibus ultor, qui face barbatos ferroque sequare Perotos? González de la Calle defendió la actitud del Brocense ante Nebrija: aunque no parece haber percibido la ironía y el buen humor del Brocense, sí tuvo muy presente su rebeldía ante la resistencia de la universidad a reemplazar la gramática de Nebrija como texto obligatorio. Ha sido José María Maestre (1989) quien, ya en nuestros días y con mucha agudeza, ha puesto de manifiesto la astucia del extremeño al demostrar cómo el prólogo a la Minerva permitió al Brocense colocarla por encima de la Gramática de Nebrija, que ya había cumplido su misión. Sin embargo, el Brocense no llegó a ver el éxito de su estrategia, ya que, mediante 
Real Cédula otorgada en Madrid el 8 de octubre de 1598, se daba al "Antonio" la exclusiva como texto obligatorio de enseñanza. En suma, este Ensayo biográfico fue un libro pionero en el esfuerzo por recuperar la obra de los humanistas españoles. Pero no fue la última palabra que González de la Calle expresara sobre esta figura, ya que en 1928 publicaría en los talleres tipográficos de la revista Archivos un nuevo libro, titulado Contribución a la biografía del Brocense.

En 1926 González de la Calle se trasladó a la Universidad Central de Madrid, donde ocupó una plaza de auxiliar temporal de Lengua y Literatura Latinas en la cátedra de Julio Cejador y Frauca (18641927), quien se acababa de jubilar. Tenía la intención de ocuparla definitivamente, pero en la convocatoria correspondiente fue excluido del concurso, precisamente por estar sobrecalificado ${ }^{2}$. No obstante esta frustración profesional, el extremeño continuó impartiendo clases de latín en la Universidad Central. Tras la jubilación de Mario Daza Campos, del que había sido profesor ayudante y colaborador, también asumió las de sánscrito. Un primer resultado de esta nueva iniciativa suya fue la publicación de Ratnavali o El collar de perlas, de CriHarsa, una obra teatral traducida directamente del sánscrito y del prácrito por Pedro Urbano González de la Calle (1934). Más tarde, González de la Calle dedicaría otros trabajos a esta temprana afición suya al sánscrito y a la cultura de la India, como son su traducción anotada del Priyadarçika y la versión completa del Rtusamhara, ambas inéditas. También debe mencionarse sus "Glosas y adiciones a una versión castellana de los tres primeros cantos del Rtusamhara de Kalidasa" (1961) y su traducción con prólogo y notas de La India y el mundo, de Sylvain Levi, que se editó en México en 1956.

Fue en los años treinta cuando en España se creó la especialidad de Filología Clásica (1932) y cuando asimismo se fundó la revista Emerita (1933), boletín de filología clásica en el que también colaboró Pedro Urbano. Este florecimiento de los estudios clásicos, al modo y semejanza de lo que ocurría en Alemania, Francia o Inglaterra, dio lugar a que se dotaran más cátedras, lo que hizo posible que Pedro Urbano retornara oficialmente a la Universidad Central en 1932. Ya para entonces había alcanzado un prestigio científico muy sólido, que

${ }^{2}$ La plaza la ocupó Bernardo Alemany Selfa (1896-1972), catedrático de la Universidad de Granada desde 1922. Latinista mucho más moderno que Pedro Urbano, Alemany Selfa tenía, en cambio, un currículum profesional sensiblemente inferior al suyo, como se reconoce en la resolución firmada por el Director General de Enseñanza Superior y Secundaria el 26 de julio de 1927: “...no pudiendo incluirse al Sr. González de la Calle, porque reuniendo méritos por su brillante carrera y excelentes y reconocidas obras publicadas para desempeñar con brillantez la Cátedra de que se trata, la Ley lo excluye para el presente concurso....". El dato proviene del expediente de González de la Calle conservado en la Sección de Personal de la Facultad de Filología de la Universidad Complutense de Madrid. Tomado de García Jurado 2009a. 
venía avalado por publicaciones y estudios como la Influencia de las literaturas clásicas en la formación de la personalidad (1920) y la traducción de la Lingüistica indoeuropea de Rudolf Meringer (1923). Con esta traducción puso a disposición de los estudiosos un manual para una materia muy cercana al sánscrito, que ya formaba parte del currículum académico de la Universidad Central ${ }^{3}$.

También datan del periodo madrileño su reseña de la Historia de la literatura de Alfred Gudemann (1927) y su traducción de la obra de Eduard Norden, Die römische Literatur: Die lateinische Literatur im Übergang vom Altertum zum Mittelalter (La literatura latina: en la transición de la Antigüedad a la Edad Media), que el autor alemán había publicado en 1910 y que tendría múltiples reediciones. Con su decisión de emprender esta traducción de la obra de Norden (que por desgracia no llegó a publicarse), González de la Calle demostraba estar al día en las nuevas corrientes lingüísticas y literarias que florecían en Europa y de modo especial en Alemania, donde el cultivo de la filología clásica tuvo un auge notable, solamente truncado por el acceso al poder del nacionalsocialismo, pues figuras como Werner Wilheim Jaeger o el propio Norden fueron víctimas del antisemitismo nazi, por lo que tuvieron que emigrar, el primero a los Estados Unidos y el segundo a Suiza (Pedro Urbano no sabía a esas alturas de su vida que iba a sufrir él mismo el duro destierro). De cualquier modo, con la traducción de Norden, González de la Calle esperaba poder poner a disposición de su círculo más cercano el pensamiento de quien también había escrito Die antique Kunstprosa von VI. Jahrhundert v. Chr. Bis in die Zeit der Renaissance (Leipzig, 1898) y que gozaba de prestigio inmenso en esos años ${ }^{4}$. También debemos comentar que en esa época madrileña, Pedro Urbano tenía preparada la traducción de la Historia de la literatura latina, de Friedrich Leo, para la colección Estudios de Emerita, tal y como se anunció en Emerita, en 1935. Sin embargo, el estallido de la Guerra Civil impidió su publicación, que no se dio hasta 1950, en Bogotá.

Al estallar la guerra, González de la Calle no dudó en ponerse de lado de la República y del gobierno legalmente constituido. La formación krausista que recibió de su padre, las ideas políticas y pedagógicas que heredó de la Institución Libre de Enseñanza, la afinidad que sentía con los hermanos Francisco y Hermenegildo Giner de los Ríos, con Manuel Bartolomé Cossío y con Nicolás Salmerón, su propio entorno familiar, y sobre todo su cabal sentido del deber y de la dignidad personal, hicieron de él un fiel partidario de la causa republicana. En noviembre de 1936, cuando comenzaron los brutales

3 Sobre la introducción y el desarrollo de esta materia en el currículum universitario, véase Álvarez Pedrosa 1994.

4 Véase Kytzler et al. 1994. 
enfrentamientos en la Ciudad Universitaria, cuyos edificios (algunos todavía sin estrenar) se convirtieron en frente de batalla, González de la Calle formó parte del grupo de profesores e intelectuales distinguidos que fue evacuado a Valencia y de allí, tiempo después, a Barcelona (véase Blasco y Mancebo 2008-09). En la Ciudad Condal dio clases de Poesía Latina e Historia de la Filología Clásica, hasta que la cercanía de los ejércitos de Franco lo obligó a marcharse. Recordaría el 23 de enero de 1939 como la fecha "en que emigré voluntariamente de mi patria”.

\section{El exilio. Colombia}

Después de abandonar España, González de la Calle se trasladó a París, donde tuvo la suerte de entrar en contacto con el entonces presidente de Colombia Eduardo Santos Montejo, quien extendió a él y a su esposa Angelita una invitación para refugiarse en Bogotá. Los trámites fueron relativamente rápidos. En carta de abril de 1939, el ministro de Educación de Colombia escribió al director de la Escuela Normal Superior sobre el caso del eminente académico, "quien desea establecerse en el país, dedicándose al magisterio, profesión que ha ejercido por muchos años y con gran éxito en Europa”. El ministro anunció enseguida al rector de la Normal Superior que lo autorizaba a ofrecerle, a su llegada, unas clases de Lingüística, y agregó: "Ud. se servirá indicar al profesor González de la Calle el sueldo del que disfrutará de acuerdo con las horas de clase que le corresponde dictar" (Silva 2013).

Según datos consulares aportados por Martínez Gorroño (2003), el matrimonio llegó a Colombia el 22 de julio de 1939. A partir de agosto de ese año, hasta diciembre de 1949, González de la Calle tuvo a su cargo las cátedras de Lingüística General, Lengua Latina, Gramática Histórica de la Lengua Castellana y Lengua Sánscrita en la Escuela Normal Superior de Bogotá. Fue, además, profesor encargado de Latín (1946-1948) en el Instituto de Filosofía y Letras de la Universidad Nacional de Colombia y profesor de Latín del mismo Instituto (posteriormente Facultad) durante el año de 1949. En 1944 fue designado por el Ministerio de Educación Nacional como "Colaborador técnico” del entonces Instituto Rufino José Cuervo, luego Instituto Caro y Cuervo, en el que trabajó hasta 1949.

Si hubo una institución educativa colombiana que acogió a los exiliados españoles, resalta Hernández García (2012), fue la Escuela Normal Superior, "La Normal”, regida por José Francisco Socarrás (1906-1995), médico psiquiatra. Este centro tenía como objetivo principal preparar a los maestros, hasta entonces profesores sin una especialización concreta, para la enseñanza en las distintas profesiones. 
Por lo mismo, el exilio español pudo contribuir a llenar las carencias que padecía el sistema educativo colombiano en comparación con el de otros países de América. Pero el proceso de inmigración fue muy selectivo: durante el gobierno de López Pumarejo (1934-1938), lo mismo que durante el de Santos Montejo (1938-1942), fueron examinadas con lupa las solicitudes de los españoles que pedían la correspondiente visa ${ }^{5}$. Excepcionalmente, algunos recibieron una invitación de los máximos dirigentes del país, como fue el caso de González de la Calle y el del pedagogo y político Luis de Zulueta. También fueron invitados por Eduardo Santos la psicóloga Mercedes Rodríguez Bellido, el químico Antonio García Banús (quien había sido catedrático de la Sorbona), el ecólogo José Cuatrecasas Asumí, el arquitecto José de Recasens, el pedagogo Miguel Fornaguera, el jurista José María Ots Capdequí, el profesor de Literatura granadino José Francisco Cirre y su esposa la arabista Manuela Manzanares (quien, además, ejerció de bibliotecaria).

De los intelectuales europeos que se refugiaron en Colombia fueron dos los profesores que lograron hacer escuela: el etnólogo francés Paul Rivet (1876-1958), quien fundó el Instituto Etnológico Nacional, donde formó un nutrido grupo de etnólogos, y nuestro Pedro Urbano, quien al llegar se hizo cargo de las cátedras de Lingüística General y Gramática Histórica del Español en la Escuela Normal, la cual sería durante algunos años el único centro universitario que ofrecía este tipo de enseñanza al alumnado. Según Gabriel Betancur Mejía y Francisco Sánchez Arévalo (1967, p. 136):

La severa y austera personalidad del profesor, su bien fundado prestigio internacional y el alto nivel científico de sus cursos, completamente inusitado en nuestro medio, produjeron profunda impresión en sus discípulos y llevaron a algunos de ellos a tomar la resolución de dedicar su vida al estudio e investigación del español. Él fue uno de los primeros en dar a conocer en la cátedra colombiana los resultados de la ciencia lingüística inaugurada por Rask y Bopp en el sector indoeuropeo y por Federico Diez en el sector románico, con especial consideración, claro está, de los logros debidos a Menéndez Pidal y su escuela en el sector hispanorromance. Incluso sus cursos de latín tenían una orientación decididamente lingüística, más que humanística.

\footnotetext{
${ }^{5}$ Fueron muy sonados los enfrentamientos de Germán Arciniegas, ministro de Educación, con Luis López Mesa, ministro de Asuntos Exteriores, que solía mostrarse muy receloso ante cualquier petición de asilo. Se decía entre los exiliados que, cuando le recomendaban alguna solicitud, López Mesa solía responder: “¿Quién me garantiza que no es un bombista anarquista o comunista?". Como ejemplo señero cita Hernández García (2012, pp. 136-137, n. 2) el caso del escritor austriaco Stefan Zweig, quien, al huir del nazismo, se exilió en Brasil y acabó suicidándose sin conseguir que Arciniegas lograra llevarlo a Colombia.
} 
En 1940, a instancias del ministro de Educación, Jorge Eliécer Gaitán, el gobierno nacional creó el Instituto Rufino José Cuervo, que en 1942 se constituyó definitivamente como el Instituto Caro y Cuervo, al que se vinculó con todo entusiasmo Pedro Urbano. Durante nueve años dio clases ininterrumpidamente. Sus cursos, destinados principalmente a los investigadores del Instituto, aunque abiertos a todo aquel que quisiera participar en ellos, fueron los siguientes: Gramática histórica española (que incluía el estudio pormenorizado del Poema del mio Cid y la discusión de una larga serie de rectificaciones, la mayor parte de carácter prosódico, que el exiliado hacía al Manual de gramática histórica española de Menéndez Pidal); Latín vulgar; Sintaxis del castellano; Sintaxis de las Glosas silenses y emilianenses; Métrica clásica; Lexicografía general; Sánscrito, y las llamadas Orientaciones doctrinales para la investigación lingüística y filológica del castellano en América; Contribución al estudio del "bogotano" y Cuestiones del castellano en Colombia.

A su actividad docente, ahora decididamente orientada al español y más específicamente al español de América, señalan Betancur Mejía y Sánchez Arévalo (1967, p. 137), "llevó también el profesor González de la Calle su elevado espíritu científico y contribuyó con sus cursos, con su trabajo y con su ejemplo a dar a las labores de esta casa de estudios el nivel que la ciencia exige". El prestigio del que venía precedido, y que se fue consolidando día a día en sus clases, hizo que en abril de 1944 fuera nombrado por decreto colaborador técnico del Instituto Caro y Cuervo ${ }^{6}$. Este nombramiento suponía un reconocimiento oficial al trabajo que venía realizando junto al Profesor-Director, el padre jesuita Félix Restrepo, sobre todo en la continuación del Diccionario de construcción y régimen de la lengua castellana de Rufino José Cuervo. Al respecto son significativas las palabras con las que abre el primer número del Boletín del Instituto el padre Félix Restrepo (1945, p. 2):

Reunidos bajo el nombre, no oficial, de Instituto Rufino J. Cuervo, y en virtud de un contrato con el Gobierno Nacional, hemos trabajado desde entonces, en horas contadas y por consiguiente con muy poca intensidad, el profesor español Pedro Urbano González de la Calle y yo, con la contribución de los señores Julián Motta Salas, Rafael Torres Quintero y Francisco Sánchez Arévalo, y con la desinteresada y gentilísima colaboración de la señora Cecilia Hernández de Mendoza.

${ }^{6}$ El Decreto 786, del 31 de marzo de 1944, recogía lo siguiente en su artículo $7^{\circ}$ : "El Profesor-Director del Instituto dará las clases de Latín y Griego a los auxiliares y asistentes que no tengan conocimiento de estas lenguas y dará a los mismos una hora diaria de clase sobre materias necesarias para que la continuación del Diccionario de construcción y régimen no decaiga del alto nivel en que lo dejó su iniciador". Por Decreto 973 , de 24 de abril de 1944, en su artículo $2^{\circ}$, se establece: "Nómbrase Colaborador técnico del mismo Instituto al señor don Pedro Urbano González de la Calle". 
El profesor González de la Calle estableció en el Instituto Rufino J. Cuervo su cátedra de sánscrito, ha dado un curso de historia del castellano y otro, del castellano en América, y ha atendido diversas consultas de investigadores y de empleados de la Biblioteca Nacional.

Por esas fechas, González de la Calle se dedicó preferentemente a estudiar el castellano, la dialectología del español de América y, sobre todo, la obra inacabada del gran Rufino José Cuervo ${ }^{7}$. De este periodo datan las siguientes publicaciones suyas: "Notas para una posible adición a un texto del maestro Cuervo" (1941); "Breve comentario a unas líneas inéditas del maestro Cuervo" (1944); "Bibliografía de la obra Castellano popular y castellano literario", en Obras inéditas de Rufino J. Cuervo (1944); "Consideraciones acerca de la significación científica de la labor lingüística del maestro Cuervo" (1944); "Orientaciones doctrinales para la investigación lingüística y filológica del castellano en América" (1944); "Concepto de un diccionario de construcción y régimen: anotaciones lexicológicas" (1945); "Formación general lingüística del maestro don Rufino J. Cuervo. Apuntes para un ensayo" (1945); "De re lexicographica: notas y comentarios" (1945); "Glosa a un texto dudoso del maestro Cuervo (notas de lectura)" (1946); “Admoniciones para el estudio de la gramática histórica del castellano o español" (1946) y alguna otra que fue apareciendo después de haberse marchado el filólogo de Colombia, como veremos en su momento.

Pedro Urbano se dedicó en estos años a resaltar las peculiaridades del español de América y particularmente del bogotano, variedades lingüísticas por las que sentía "suma simpatía y comprensión" (Betancur Mejía y Sánchez Arévalo 1967, p. 139). Ello le valió la crítica incomprensiva del hispanista holandés Jonas A. van Praag, quien, escandalizado por su Contribución al estudio del bogotano, acusó a los españoles que trabajaban en América de tratar de congraciarse con los habitantes del país donde residían. En el caso de Pedro Urbano no había nada más alejado de la realidad. Y es que el holandés estaba muy poco al corriente de lo que ocurría no sólo en Colombia, sino también en otros países americanos, donde los estudios lingüísticos más avanzados ya reconocían la importancia de las variedades nacionales. Eran célebres el ensayo de J.L. Borges sobre "El idioma de los argentinos" (1927) y su ruidosa polémica con Américo Castro, que defendía el canon centralista de Madrid (sobre este tema véase, por ejemplo, Di Tullio y Bordelois 2002). Tiempo después, un discípulo

${ }^{7}$ Rufino José Cuervo (Bogotá, 1844-París, 1911) puede considerarse el padre de la filología hispanoamericana. Sus principales obras son Gramática de la lengua latina (en colaboración con Miguel Antonio Caro), Aportaciones críticas sobre el lenguaje bogotano y el Diccionario de construcción y régimen de la lengua castellana. Para decirlo con palabras de Martín Alonso (1973), Cuervo fue la figura más eminente de la filología hispánica hasta la aparición de Ramón Menéndez Pidal. 
de Menéndez Pidal, Amado Alonso, puso en marcha la Biblioteca de Dialectología Hispanoamericana, una de varias instituciones destinadas a destacar el valor del español en el continente americano. Otro tanto ocurría en otros países: en Chile, a partir de las aportaciones de Rodolfo Lenz; y en Colombia, en parte gracias a los esfuerzos de González de la Calle.

Para un filólogo clásico, como lo fue en el fondo Pedro Urbano, la lexicografía tenía una importancia muy especial. Así lo reconoció al rememorar sus años de estudiante (1951, p. 245):

En plena juventud, actuando de opositor a cátedra de lengua y literatura latinas, no pude utilizar más que léxicos muy deficientes, los que tenían general aplicación más que aceptación en el ambiente universitario no muy depurado de mis años mozos. Ni pude ni tuve tiempo de hacer una preparación sólida profesional, ni me libré de las funestas improvisaciones, que en la época que evoco estaban a la orden del día. Sólo recuerdo de esa época nada venturosa de mi vida discente que uno de mis coopositores, de memoria casi patológica, trataba de aprenderse ad pedem litterae el diccionario latino-español de D. Raimundo de $\mathrm{Miguel}^{8}$.

En esta reflexión sobre una parcela tan importante de la filología, y más todavía de la filología clásica, como lo es la lexicografía, González de la Calle no sólo denunció los diccionarios “plúmbeos, farragosos y obscuros" que tuvo que padecer, sino que también hizo un encendido elogio del diccionario latino-alemán de F.A. Heinichen (González de la Calle 1951, p. 246). Confesó que lo había consultado con gusto a lo largo de tres décadas de vida profesional sin encontrar una sola falla, lo cual contrastó con el centenar de rectificaciones que se sintió obligado a hacer en las notaciones prosódicas de la Gramática histórica española de Menéndez Pidal (se refería a la octava edición de la obra, de 1941). Recordó cómo, en los años en que trabajaba en la Universidad Central, le insistió el decano de la Facultad, Manuel García Morente, que asumiera la responsabilidad de elaborar un diccionario latino-español, pero las desventuras de la Guerra Civil frustraron tan noble propósito. Sin embargo, el destino sí le reservaba otra responsabilidad en el campo de la lexicografía. Nos lo cuenta él mismo con estas desgarradoras palabras (1951, p. 249):

El triste y necesario alejamiento de la patria querida y doliente me trajo a este hidalgo solar colombiano, en el que he llenado ya muchas páginas de mi diario de gratitud entrañada, cordialísima. Pues bien, al año de residir en Colombia, de nuevo me vi en transcendentales y abrumado-

${ }^{8}$ Esta publicación data de la etapa mexicana del autor, pero todo indica que González de la Calle escribió el texto en Colombia hacia finales de los años 40. De cualquier modo, se trata de una reflexión hecha en plena madurez. 
ras relaciones con las labores lexicográficas: como colaborador, primero, del Instituto Rufino J. Cuervo y como colaborador también después del Instituto Caro y Cuervo, he contraído el compromiso de honor de colaborar en las tareas precisas para trazar la continuación del Diccionario de construcción y régimen, comenzado por el insigne D. Rufino J. Cuervo, de sagrada y perdurable memoria.

Así, pues, González de la Calle emprendió la tarea reparando en que, como Heinichen al escribir el suyo, el lingüista bogotano trazó un luminoso proemio para su diccionario, que servía para situar la labor lexicológica en el contexto científico que el autor juzgaba más razonable, más legítimo y, por ende, más eficaz. Como afirmación inicial, nuestro autor quiso subrayar que

el más genial lexicógrafo colombiano y uno de los más autorizados del mundo culto, D. Rufino J. Cuervo, abrió ruta y dejó huella en la lexicografía sintáctica del castellano, orientando y cumpliendo su labor específica lexicológica y lexicográfica dentro de una severa, lúcida y progresiva técnica científica (1951, p. 250).

González de la Calle pasó luego a analizar las aseveraciones y conjeturas de Cuervo y la valoración que éste había hecho de los neogramáticos y de Hugo Schuchardt y su Ueber die Lautgesetze. Gegen die Junggrammatiker (1885). Cita ésta que corrobora el dominio que tenía de la bibliografía lingüística de su tiempo, como el propio Pedro Urbano puso de relieve en 1945. Pero, como no podía ser menos, en el gran trabajo de Cuervo no todo eran aciertos, de modo que a título de specimina, señaló también "ligerísimas inexactitudes" (1951, p. 259). Aparte de términos concretos, le atribuyó una indebida subestimación del elemento griego en el léxico del romance, que desmontó apoyándose en Menéndez Pidal (1951, p. 262). Asimismo, González de la Calle detectó yerros parciales en textos en que Cuervo trataba de combatir falsas valoraciones de la lengua sánscrita. De cualquier modo, espíritu generoso donde los haya, Pedro Urbano, al hacer estas rectificaciones, rindió homenaje de admiración a la ingente obra del bogotano:

Mi cordial, sentida y efusiva admiración por el insigne maestro queda enraizada y acendrada con estas muy humildes y sinceras rectificaciones, en las que intento rendir el tributo de mi honda devoción al sabio bogotano, procurando seguir su ejemplo, dentro de los estrechos límites de mi capacidad personal. He creído y sigo creyendo que, en el caso de referencia, una exaltación fetichista e irrazonable puede y debe ser sustituida por una admiración tan honda como objetiva y razonada. Y así, en las parciales y esporádicas fallas de la ingente labor del maestro me ha sido posible destacar cada día con mayor relieve los considera- 
bles y numerosos aciertos de semejante creación. Creación que tiene hasta la ventaja de transformarse en eficaz estímulo de nuevas inquietudes y de nuevas tareas de abnegada investigación científica. En esta etapa final de mi ya larga existencia he hallado pues en la lexicografía del maestro Cuervo inolvidables enseñanzas y necesarios acicates para continuar en la brecha, donde siempre habrá labor que cumplir y dudas que atenuar o disipar. Afortunadamente, como ya he tenido que reconocer en otra ocasión, los grandes maestros no lo hicieron todo y nos han dejado a los humildes continuadores modesta mies que recoger y que entrojar (1951, p. 269).

Pedro Urbano terminó este importante trabajo felicitándose de la aparición en el momento de redactarlo de una obra que llevaba el título siguiente: Vox. Diccionario general ilustrado de la lengua española, prólogo de D. Ramón Menéndez Pidal y revisión de D. Samuel Gili Gaya..., porque estaba inspirado en los principios que él mismo había postulado. Especial mención le mereció el prólogo del maestro Menéndez Pidal, del que afirmó: "es tan sobrio como jugoso y denso; es, en suma, digno de la merecida fama de su ilustre autor" (1951, p. 271). Resulta curioso que en la Introducción a la lexicografía moderna que publicó Julio Casares en 1969, con prólogo de W. von Wartburg, se haya citado a Cuervo una sola vez, y entonces, de forma anecdótica, por lo que no nos resistimos a la tentación de reproducir la cita:

Cuentan que Cuervo se amargó los últimos años de su vida pensando que el ingente trabajo que hubo de realizar para su inestimable Diccionario de construcción y régimen, tenía por base en gran proporción textos indignos de la confianza que puso en ellos. Precisamente en estos días se han publicado por primera vez unas cuartillas del insigne filólogo colombiano, que podrían titularse "advertencias para lexicógrafos principiantes" y en ellas se da el grito de alarma contra las graves acechanzas con que nos amenazan a derecha e izquierda textos mendaces y editores incompetentes.

El matrimonio de Angelita y Pedro Urbano tenía la vida encauzada en Bogotá, pero los sucesos de 1948, conocidos como "el bogotazo", sin duda influyeron en sus espíritus sensibles ${ }^{9}$. Sobre todo, cierta sensación de soledad al no tener hijos ni parientes en Colombia, les llevó a tomar la determinación de trasladarse a México. Es más que probable que su hermana Dorotea (casada con Francisco Barnés), así como el entorno de José Giral, les reclamaran. Además, el ambiente político de México era totalmente favorable al exilio republicano español. Todo ello influyó en la decisión, toda vez que el filólogo tenía descartado volver a España. Atrás, en su patria, quedaba su con-

${ }^{9}$ Me refiero a la violenta reacción popular desatada por el asesinato del político Jorge Eliécer Gaitán en abril de 1948. 
dena por el Tribunal de Responsabilidades Políticas que, con fecha del 30 de enero de 1942, le inhabilitó para ejercer cargos públicos por un periodo de ocho años y le sancionó con la cantidad de veinticinco mil pesetas. Sea como fuere, Pedro Urbano se trasladó a la ciudad de México en diciembre de 1949.

\section{Tercera etapa. MÉxico}

González de la Calle inició una nueva etapa de su vida cuando, en febrero de 1950, a los setenta años, fue nombrado investigador de El Colegio de México. No tardó en colaborar con cursos monográficos sobre las fuentes de las obras de Quevedo y con una clase semanal de dos horas de sánscrito, iniciada en octubre de 1950. En los últimos meses de este mismo año también dio clases (gratuitas) en la Universidad Nacional Autónoma de México:

y esta Alma mater me contó entre sus profesores el año 1951 y me confirió en el siguiente representación y categoría de profesor ordinario de lingüística para regentar la cátedra de Lingüística General e Indoeuropea que figura en los cuadros de enseñanza de la Facultad de Filosofía y Letras de la UNAM en la sección especial de Letras Clásicas ${ }^{10}$.

En 1963 fue nombrado titular de Filología Románica y Lingüística General e Indoeuropea. Finalmente, el 31 de enero de 1964, cuando estaba a punto de cumplir los 85 , causó baja por jubilación.

La vida en México era más entretenida y amena que en Colombia: los actos políticos y de afianzamiento de la República eran frecuentes. Así, tenemos varios testimonios de su asistencia a actos como el organizado por el Ateneo en homenaje a Unamuno en 1961. Lógicamente, en esta tercera etapa de su vida la actividad docente fue menos intensa y las publicaciones, menos numerosas. Todavía en 1955 publicó cuatro trabajos relacionados con Cuervo, como son su "Contribución al estudio del bogotano" (1955), "Cuervo en Europa” (1959), "Una edición de las Disquisiciones sobre filología castellana de Cuervo" (1960) y Contribución al estudio del bogotano: orientaciones metodológicas para la investigación del castellano en América (1963). Se trata, sin duda, de trabajos que Pedro Urbano había iniciado en Colombia.

Las últimas publicaciones suyas de las que tenemos constancia incluyen un libro titulado Quevedo y los dos Sénecas, publicado por El

10 Citado por Blasco y Mancebo 2008-09, p. 185. Blasco y Mancebo han revisado el expediente personal de González de la Calle, número 10092, en la UNAM, que contiene el curriculum vitae presentado el 30 de noviembre de 1962. Allí consta que estaba casado y que vivía domiciliado en la calle Amazonas 26, en la colonia Cuauhtémoc del Distrito Federal. 
Colegio de México en 1965, en el que González de la Calle acreditó lo mucho que el autor español debía a los dos autores clásicos, mediante los cuales llegaba a las fuentes griegas. En un breve prefacio anónimo se hace constar que con esta publicación "El Colegio de México rinde un homenaje de afecto y gratitud a su autor". También se señala lo siguiente:

Don Pedro Urbano vino al Colegio en 1950, y en ese mismo año, además de iniciar sus clases de sánscrito (que continúan hasta la fecha) dio un curso sobre "Humanistas españoles de los siglos XVI y XVıI". Raimundo Lida, director a la sazón del Centro de Filología, solía hacerle consultas sobre textos de Quevedo mal descifrados o mal impresos en las ediciones corrientes. Las respuestas de Don Pedro Urbano, largamente meditadas, ocupaban a veces cuarenta cuartillas. Trabajo callado, silencioso, abnegado. Trabajo heroico, también, a causa de la penuria mexicana en libros antiguos de humanidades clásicas. Esas respuestas constituyen, básicamente, la materia del presente libro (González de la Calle 1965, p. 7).

Entre los últimos textos de González de la Calle también cabe destacar una conferencia presentada en la Facultad de Filosofía y Letras el 17 de octubre de 1960. Según parece, tuvo que ser leída por una discípula, la señorita Leticia Fragoso, porque el propio autor no se sentía con fuerzas para hacerlo. El texto comienza con estas palabras: "Excusen los oyentes la presencia «física» del autor de estas líneas, abrumado de años y flaquezas, y reciban su respetuosa salutación”. La conferencia, que se imprimiría póstumamente en Nova Tellvs. Anuario del Centro de Estudios Clásicos de la UNAM, llevaba por título "Desiderata de las investigaciones acerca del humanismo español". Con ella, Pedro Urbano terminó su larga andadura profesional como la empezó, dedicando su atención al humanismo español: "Una modesta, aunque ya antigua afición a los estudios referentes a la historia del humanismo español, nos ha permitido cosechar algunos humildes frutos, que quisiéramos saber ofrendar aquí” (1985, p. 149). El filólogo terminó su alocución con esta reflexión general, que era resumen de su propia actitud moral:

El "hombre" ha de poner sus mejores esencias humanas en la profesión que abnegada y eficazmente ejerza, y el "profesional" no olvidará nunca el soporte humano esencial, radical de su especialización técnica o científica. Pocas disciplinas permiten formar y mantener la inescindible síntesis del "hombre" con el "profesional" como las disciplinas calificadamente humanísticas (p. 184). 


\section{Conclusión}

Estas páginas han querido llamar la atención sobre una obra ingente y una actitud ejemplar. Desde el primer momento de la contienda civil, Pedro Urbano González de la Calle optó por la dignidad del exilio antes que por una vida próspera; por un destierro que, aun con el agradecimiento que demostró tener por la gran hospitalidad y generosidad de los países acogedores, siempre le hizo sentir un extraño. Eso explica el que, pese a los honores académicos que recibiera en Colombia, fuera a México en busca del apoyo familiar cuando ya había cumplido los setenta años. Siempre añoraba España. "Su figura humanista", según recordaría Cecilia Hernández de Mendoza, "parecía arrancada a un cuadro del Greco: su barba entrecana hacía contraste con la expresión infantil y asombrada de los ojos a los cuales día tras día se asomaba un nuevo descubrimiento". En esta nota necrológica, publicada en el diario El Tiempo de Bogotá el 12 de marzo de 1967, Hernández de Mendoza también señaló lo siguiente:

Para quien siguió sus sabias enseñanzas; para quien el docto maestro fue ejemplo y permanente guía; para quien guarda con devoción y gratitud muchos borradores de sus trabajos en Colombia, la figura del profesor González de la Calle, al perderse en la bruma de lo eterno, se hace en el tiempo presente estatua imperecedera... Guarde su nombre la España inmortal de sus recuerdos; guárdelo El Colegio de México y la Universidad Autónoma de este país; Colombia habrá de guardarlo en el Instituto Caro y Cuervo, en cada uno de sus alumnos y en la más alta tradición de su cultura.

\section{REFERENCIAS}

Alonso Pedraz, Martín 1973. Ciencia del lenguaje y arte del estilo, Aguilar, Madrid.

Álvarez Pedrosa, J.A. 1994. "La lingüística indoeuropea en España hasta 1930", Revista Española de Lingüística, 24, 1, pp. 49-67.

Betancur Mejía, Gabriel y F. Sánchez Arévalo 1967. "Pedro Urbano González de la Calle", Thesaurus. Boletín del Instituto Caro y Cuervo, 22, pp. 131-146.

Blasco Gil, Yolanda y M. Fernanda Mancebo 2008-09. "Niceto Alcalá-Zamora y Pedro Urbano González de la Calle. Profesores exilados y provisión de sus cátedras", Cuestiones Pedagógicas, 19, pp. 173-189.

Casares, Julio 1969. Introducción a la lexicografía moderna, CSIC, Madrid. (Anejos de la Revista de Filología Española, 52).

Di Tullio, Ángela e Ivonne Bordelois 2002. "El idioma de los Argentinos: cultura y discriminación”, Ciberletras. Revista de Crítica Literaria y de Cultura, 6.

GAos, José 1999. Obras Completas. T. 19: Epistolario y papeles privados. Ed. Alfonso Rangel Guerra, Universidad Nacional Autónoma de México, México.

García Jurado, Francisco 2008. "El nacimiento de la filología clásica en España. La Facultad de Filosofía y Letras de Madrid (1932-1936)”, Estudios Clásicos, 50, pp. $77-104$. 
García Jurado, Francisco 2008a. "Acerca de Eduard Norden", http:/ lectoresaudaces. blogspot.mx/ search?q =norden [consultado el 7 de enero de 2016].

García Jurado, Francisco 2009. "Cuando el tiempo se detiene. Los avatares de una historia de la literatura latina publicada en Colombia: Pedro Urbano González de la Calle", Literatura: Teoría, Historia, Crítica, 11, pp. 303-332.

García Jurado, Francisco 2009a. "Ejemplo y memoria de Pedro Urbano González de la Calle", http:/ / lectoresaudaces. blogspot.mx / 2009/ 01 / ejemplo-y-memoria-de-pedro-urbano.html [consultado el 30 de diciembre de 2015].

González de la Calle, P.U. 1922. Vida profesional y académica de Francisco Sánchez de las Brozas, Librería General de Victoriano Suárez, Madrid.

González de la CAlle, P.U. 1945. "Formación general lingüística del maestro don Rufino J. Cuervo. Apuntes para un ensayo", Thesaurus. Boletín del Instituto Caro y Cuervo, 1, pp. 212-241.

González de la Calle, P.U. 1948. "Francisco de Vergara y la pronunciación de la $z$ griega. Apuntes para una monografía", Thesaurus. Boletín del Instituto Caro y Cuervo, 4, pp. 249-320.

González de la Calle, P.U. 1951. "Experiencias lexicográficas. Apuntes y notas", Thesaurus. Boletín del Instituto Caro y Cuervo, 7, pp. 245-279.

González de la Calle, P.U. 1965. Quevedo y los dos Sénecas, El Colegio de México, México.

Hernández García, José Ángel 2012. "La influencia pedagógica del exilio republicano español: la edad de oro de la enseñanza en Colombia", Latinoamérica, 1, pp. 135-153.

Kytzler, Bernhard, Kurt Rudolph und Jörg RüPke (Hrsg.) 1994. Eduard Norden (1868-1941). Ein deutscher Gelehrter jüdischer Herkunft, Franz Steiner Verlag, Stuttgart. (Palingenesia, 49).

Maestre Maestre, J.M. 1989. "Barbatos Perotos: los tópicos del prólogo-dedicatoria de la Minerva", en Actas del Simposio Internacional IV Centenario de la publicación de la Minerva del Brocense: 1587-1987, Institución Cultural El Brocense-Diputación Provincial, Cáceres, pp. 203-232.

Martínez Gorroño, M.E. 2003. "La aportación que supuso para Colombia el trabajo de Pedro Urbano González de la Calle", Actas del Congreso Internacional celebrado en el marco del Congreso Plural: Sesenta años después (Madrid-Alcalá-Toledo, diciembre de 1999). Coords. Manuel Lluisa y Alicia Alted Vigil, UNED, Madrid, t. 1 , pp. $189-198$.

Restrepo, Félix 1945. "Para la historia", Thesaurus. Boletín del Instituto Caro y Cuervo, 1, pp. 1-10. 


\section{APÉNDICE BIBLIOGRÁFICO \\ LISTA DE LAS PRINCIPALES PUBLICACIONES \\ DE PEDRO URBANO GONZÁLEZ DE LA CALLE}

Monografías

Sebastián Fox Morcillo: estudio histórico-crítico de sus doctrinas, Imprenta del Asilo de Huérfanos del Sagrado Corazón de Jesús, Madrid, 1903.

Francisco Sánchez de las Brozas "El Brocense", Imp. y Lib. de Francisco Núñez, Salamanca, 1912.

Varia. Notas y apuntes sobre temas de letras clásicas, Librería General de Victoriano Suárez, Madrid, 1916.

Vida profesional y académica de Francisco Sánchez de las Brozas. Ensayo biográfico, Librería General de Victoriano Suárez, Madrid, 1922.

Consideraciones acerca de la $2^{a}$ Paradoja de "El Brocense", Talleres Tipográficos de Extremadura, Cáceres, 1924.

Contribución a la biografía del Brocense, Tipografía de Archivos, Madrid, 1928.

Constituciones y bulas complementarias dadas a la Universidad de Salamanca por el Pontífice Benedicto XIII (Pedro de Luna). Edición paleográfica con prólogo y notas de Pedro Urbano González de la Calle y Amalio Huarte y Echenique, Revista Universidad, Zaragoza, 1932.

Contribución al estudio del "bogotano". Orientaciones metodológicas para la investigación del castellano en América, Publicaciones del Instituto Caro y Cuervo, Bogotá, 1963. (Series Minor, 9).

Quevedo y los dos Sénecas, El Colegio de México, México, 1965.

\section{Artí́culos}

"Causas teleológicas del acento (notas y observaciones)", Anales de la Asociación Española para el Progreso de las Ciencias. Congreso de Madrid: 15-20 de junio 1913, Madrid, 1913.

"Del amor a la verdad. Apuntes y notas", Anales de la Asociación Española para el Progreso de las Ciencias. Congreso de Madrid: 15-20 de junio 1913, Madrid, 1913.

"Ideas económicas del filósofo hispalense Sebastián Fox Morcillo", Revista de Archivos, Bibliotecas y Museos, 29 (1913), pp. 217-224.

"Ideas político-morales del P. Juan de Mariana", Revista de Archivos, Bibliotecas y Museos, 29 (1913), pp. 388-406; 30 (1914), pp. 46-60, 201-228; 31 (1915), pp. 242-262; 32 (1916), pp. 400-419.

"Análisis métrico del Carmen LXIII de Catulo", Revista de Archivos, Bibliotecas y Museos, 34 (1916), pp. 58-79.

"Influencia de las literaturas clásicas en la formación de la personalidad", Revista de Archivos, Bibliotecas y Museos, 41 (1920), pp. 205-232.

"Escarceos lingüísticos", Revista de Archivos, Bibliotecas y Museos, 41 (1920), pp. 529-538.

"La Paradoja Segunda del Brocense: apuntes y notas", Arqivo de História e Bibliografia, Imprensa da Universidade, Coimbra, 1924.

"Documentos inéditos acerca del uso de la lengua vulgar en los libros espirituales", Boletín de la Real Academia Española, 12 (1925), pp. 76-88, 194-208, 302-317; 13 (1926), pp. 258-273, 470-497, 652-673.

"Arias Montano, humanista. Apuntes y notas para un ensayo", Revista del Centro de Estudios Extremeños, 2 (1928), pp. 17-170. 
"Contribución a la biografía de Manuel da Costa «Doctor Subtilis»", en Miscelânea de Estudos em honra de D. Carolina Michaëlis de Vasconcellos, professora da Faculdade de Letras da Universidade de Coimbra, Imprensa da Universidade, Coimbra, 1930, pp. 310-373.

"Contribución al estudio de la primera versión castellana de la Eneida", Anales de la Universidad de Madrid (Letras), 2 (1933) pp. 131-157, 259-284; 3 (1934), pp. 1-20.

"Oposiciones a cátedras en la Universidad de Salamanca durante el primer decenio de la segunda mitad del siglo XVI (1550 a 1560). Ensayo monográfico”, Erudición Ibero-Ultramarina, 4 (1933), pp. 185-249, 462-501; 5 (1934), pp. 39-75, 231268, 378-478.

"El poeta aragonés Juan Sobrarias”, Revista Zurita, 1 (1933), pp. 335-364; 2 (1934), pp. 23-68.

"Algunas observaciones acerca de la prosa de Marcial (notas para un ensayo)", Emerita, 3 (1935), pp. 1-27.

"De re metrica horatiana", Emerita, 4 (1936), pp. 38-73; pp. 248-275.

"Luis Vives y España”, Revista de Indias, 2 época, 16 (1940), p. 43.

"Notas para una posible adición a un texto del maestro Cuervo", Educación, 2a época, 2/3 (1941), pp. 202-210.

"Recuerdos personales de la vida profesional del maestro Unamuno", Revista Hispánica Moderna, 7 (1941), pp. 235-242.

"Glosa a una cita clásica”, Educación, 4 (1942), pp. 353-356.

"Nicolás Salmerón”, España (Bogotá), núms. 3/5 (1942).

"Bibliografía de la obra Castellano popular y castellano literario", en Obras inéditas de Rufino J. Cuervo, Publicaciones del Instituto Caro y Cuervo, Bogotá, 1944, pp. xix-xciv.

"Breve comentario a unas líneas inéditas del maestro Cuervo", Revista de las Indias, $70 / 71$ (1944), pp. 149-154.

"Consideraciones acerca de la significación científica de la labor lingüística del maestro Cuervo", Revista de Indias, 62 (1944), pp. 11-40.

"Orientaciones doctrinales para la investigación lingüística y filológica del castellano en América”, Universidad Nacional de Colombia, 1 (1944), pp. 25-79.

"Concepto de un diccionario de construcción y régimen: anotaciones lexicográficas", Thesaurus. Boletín del Instituto Caro y Cuervo, 1 (1945), pp. 476-492.

"De re lexicographica: notas y comentarios", Boletín de la Academia Argentina de Letras, 52 (1945), pp. 491-503.

“Don Pedro Dorado y Montero”, Revista América, 6 (1945), pp. 453-458.

"Elio Antonio de Lebrija (Aelius Antonius Nebrissensis): notas para un bosquejo biográfico", Thesaurus. Boletín del Instituto Caro y Cuervo, 1 (1945), pp. 80-129.

"Formación general lingüística del maestro don Rufino J. Cuervo. Apuntes para un ensayo" Thesaurus. Boletín del Instituto Caro y Cuervo, 1 (1945), pp. 212-241.

"Adiciones lexicológicas", Thesaurus. Boletín del Instituto Caro y Cuervo, 2 (1946), pp. 12-38.

"Camino a seguir, trabajo a realizar", Thesaurus. Boletín del Instituto Caro y Cuervo, 2 (1946), pp. 535-546.

"Glosa a un texto dudoso del maestro Cuervo (notas de lectura)", Boletín de la Academia Argentina de Letras, 55 (1946), pp. 193-201.

"Orientación filológica de Leibnitz. Apuntes trazados con ocasión del tercer centenario del nacimiento de dicho filósofo", Thesaurus. Boletín del Instituto Caro y Cuervo, 2 (1946), pp. 233-276.

"Admoniciones para el estudio de la gramática histórica del castellano o español”, Boletín de la Academia Venezolana, 51 (1946), pp. 331-355; Universidad Nacional de Colombia, 10 (1947), pp. 9-27.

"Aclaraciones a un texto del autor", Thesaurus. Boletín del Instituto Caro y Cuervo, 4 (1948), pp. 572-580. 
"Francisco de Vergara y la pronunciación de la $z$ griega. Apuntes para una monografía”, Thesaurus. Boletín del Instituto Caro y Cuervo, 4 (1948), pp. 239-320.

"Glosa a unas glosas", Revista de la Universidad Nacional, 12 (1948), pp. 9-31.

"Advertencias al margen de una etimología griega de una palabra castellana. (Notas para una monografía)", Thesaurus. Boletín del Instituto Caro y Cuervo, 5 (1949), pp. 101-111.

"Anotaciones a un texto magistral”, Miscelânea de filología, literatura e história cultural à memória de Francisco Adolfo Coelho (1847-1919), Centro de Estudios Filológicos, Lisboa, 1950, t. 2, pp. 41-54.

"«Lo compramos con él» (glosas a una construcción consultada)", Thesaurus. Boletín del Instituto Caro y Cuervo, 6 (1950), pp. 282-292.

"Experiencias lexicográficas: apuntes y notas", Thesaurus. Boletín del Instituto Caro y Cuervo, 7 (1951), pp. 245-279.

"Cómo citaban a veces los humanistas y... cómo no se debe citar. Divagaciones humanísticas", Filosofía y Letras, 57/58/59, enero-diciembre de 1955, pp. 215-249.

"Contribución al estudio del «bogotano»", Revista Nacional de Cultura, 51 (1955), pp. 38-54.

"Cuervo en Europa", Nivel (México), 3 (1959), pp. 1-2.

"Personalidad lingüística del idioma catalán", Thesaurus. Boletín del Instituto Caro y Cuervo, 14 (1959), pp. 1-19.

"Una edición de las Disquisiciones sobre filología castellana de Cuervo", Thesaurus. Boletín del Instituto Caro y Cuervo, 15 (1960), pp. 276-293.

"Glosas y adiciones a una versión castellana de los tres primeros cantos del Rtusamhara de Kalidasa", Anuario de Letras, 1 (1961), pp. 13-31; 2 (1962), pp. 119-156; 3 (1963), pp. 191-218.

"Desiderata de las investigaciones acerca del humanismo español", Nova Tellvs. Anuario del Centro de Estudios Clásicos (Universidad Nacional Autónoma de México), 3 (1985), pp. 149-185.

\section{Traducciones}

Rudolf Meringer, Lingüistica indoeuropea. Traducción directa, introducción y notas de Pedro Urbano González de la Calle, Librería General Victoriano Suárez, Madrid, 1923.

Criharsa, Ratnavali o el Collar de perlas. Comedia traducida directamente del sánscrito y prácritos por Pedro Urbano González de la Calle. Introducción al estudio de la dramática india antigua por Mario Daza Campos, Imprenta de Victoriano Suárez, Madrid, 1934.

Friedrich Leo, Literatura romana. Traducción de Pedro Urbano González de la Calle, Publicaciones del Instituto Caro y Cuervo, Bogotá, 1950.

Sylvain Levi, La India y el mundo. Traducción, prólogo y notas de Pedro Urbano González de la Calle, Universidad Nacional Autónoma de México, México, 1956.

[Hay noticias de que González de la Calle realizara también los siguientes trabajos: una traducción de La literatura latina en la transición de la Antigüedad a la Edad Media, de Eduard Norden, una traducción anotada del Priyadarçika y una versión completa del Rtusamhara. Ninguna de estas traducciones parece haberse publicado y no se sabe si se conserve algún manuscrito relacionado con ellas]. 
RESEÑAS

Sobre Jean Baruzi, Saint Jean de la Croix et le probléme de l'experience mystique, en Boletín de la Real Academia de la Historia, 87 (1925), pp. 298-305.

Sobre Alfred Gudemann, Historia de la literatura latina, traducción de Carles Riba, en Revista de Filología Española, 14 (1927), pp. 294-297.

Sobre Vincenzo Ussani, Storia della letteratura latina dalle origini al 14 d.C., en Emerita, 3 (1935), pp. 376-378.

Sobre Antonio Tovar, Gramática histórica latina: sintaxis, en Thesaurus. Boletín del Instituto Caro y Cuervo, 4 (1948), pp. 164-184.

Sobre Charles Bally, Manuel de accentuation grecque, en Thesaurus. Boletín del Instituto Caro y Cuervo, 7 (1951), pp. 403-407.

Trabajos CONSERVAdos inéditos EN la Biblioteca

de El Colegio de México

En su conferencia "Desiderata de las investigaciones acerca del humanismo español", publicada póstumamente en 1985, pero cuya lectura debió hacerse antes de 1965, González de la Calle señaló, entre otras cosas, lo siguiente: "Llevo además diez años estudiando el humanismo clásico y cristiano en algunas de las obras eruditas de don Francisco de Quevedo y Villegas: hasta la fecha en que trazo estas líneas, mis investigaciones humanísticas sobre Quevedo me han permitido trazar las monografías cuyos rótulos transcribo a continuación: I. Comentarios al epistolario completo de Quevedo; II. Comentarios al supuesto Epistolario apócrifo del mismo Quevedo; III. Comentarios a las versiones de Epístolas de Séneca y de Plinio por Quevedo; IV. Comentarios a unas notas autógrafas de Quevedo al Epistolario de Séneca a Lucilio; V. Comentarios al tratado de Quevedo acerca de la Providencia de Dios; VI. Comentarios acerca de las fuentes clásicas y cristianas utilizadas por Quevedo en su opúsculo: «Nombre, origen, intento, recomendación y descendencia de la doctrina estoica. Defiéndese Epicuro de las leyendas vulgares»; VII. Quevedo, intérprete y continuador de Séneca el Retórico; VIII. Quevedo, intérprete del tratado pseudo-«senequiano» De remediis fortuitorum [este título y el anterior forman parte de Quevedo y los dos Sénecas, de 1965]; y IX. Comentarios a la «Vida de San Pablo Apóstol» trazada por don Francisco de Quevedo y Villegas” (p. 169). Señaló asimismo que estas nueve monografías sobre Quevedo eran propiedad de El Colegio de México, que en ese momento no había resuelto aún "si se deberán mandar a la imprenta” (p. 170).

En la Biblioteca Daniel Cosío Villegas de El Colegio de México se conservan inéditos los siguientes trabajos:

Comentarios a la "Vida de San Pablo Apóstol" trazada por Don Francisco de Quevedo. Manuscrito mecanografiado y encuadernado, sin fecha. Donación del autor: 10 de junio de 1966,184 pp. (primera parte) + 132 pp. (segunda parte) + 11 pp. (apéndice). [Clasificación: 867.3 G6431c].

Glosas al supuesto epistolario apócrifo de Quevedo. Manuscrito mecanografiado y encuadernado, sin fecha. Donación del autor: 10 de junio de 1966, 114 pp. (estudio) + 31 pp. (notas) + 20 pp. (apéndice). [Clasificación: 867.3 G6431gl].

Estoicos y epicúreos. Manuscrito mecanografiado y encuadernado, sin fecha. Donación del autor: 13 de junio de 1966, 114 pp. [Clasificación: 188 G6431e].

Glosas al tratado de Quevedo titulado: "Providencia de Dios". Manuscrito mecanografiado y encuadernado, sin fecha. Donación del autor: 10 de junio de 1966, 366 pp. [Clasificación: 867.3 G6431g]. 